\title{
Image Registration by Simulating Human Vision
}

\author{
Shubin Zhao \\ Jiangsu Automation Research Institute, \\ 42 East Hailian Road, Lianyungang, Jiangsu, China 222006 \\ zhao_shubin@163.com
}

\begin{abstract}
In this paper, an efficient and robust algorithm is proposed for image registration, where the images have been acquired at different times, by different sensors and some changes may take place in the scene during the time interval when the images were taken. By simulating human vision behaviors, image registration is carried out through a two-stage process. First, the rotation angles are computed by comparing the distributions of gradient orientations, which is implemented by a simple 1-D correlation. Then, a new approach is presented to detect significant corners in two images and the correspondences are established between corners in two images. At this time, the orientation difference has been removed between the images, so the corner correspondences can be established more efficiently. To account for the false corner correspondences, the voting method is used to determine the transformation parameters. Experimental results are also given.
\end{abstract}

Keywords: image registration, human vision, corner detection.

\section{Introduction}

Image registration is the process of spatially registering two or more images of the same scene, taken at different times, from different viewpoints, and/or by different sensors. It is a critical step in many applications of image analysis and computer vision, such as image fusion, change detection, video geo-registration, pattern and target localization, and so on. Because of its importance in various application areas and its complicated nature, image registration has been the topic of much recent research. During the last decades, many kinds of approaches have been proposed to address image registration problems, and the comprehensive and excellent surveys can be found in [1-2].

There are four important aspects in image registration: 1) transformation space; 2) feature space; 3) similarity measure; and 4) search strategy. Selection of transformation space is application-dependent. For example, if we know there exists affine transform between two images, affine transformation space should be selected to align the images.

According to what objects are used to align images, the approaches can be categorized into two classes: intensity-based methods and feature-based ones. Intensity-based methods directly use raw image data (intensity values) as features and the images are usually compared with cross-correlation function as similarity 
measure. Because the imaging conditions may be quite different and some changes may take place in the scene during the time interval when the images were taken, there are almost inevitably many differences between the images. Consequently, for image registration to be robust, feature-based ones are preferred, whereas intensitybased methods are usually not applicable. Most commonly used features may be significant regions [3-4], line segments [5-6], line intersections [7], and edge points [8]. How to select features depends on the given task.

Apart from feature selection, similarity measure plays an important role in image registration. Huttenlocher et al. compare images transformed by translation or translation plus rotation, where edge points are used as matching features and the Hausdorff distance is adopted to measure the similarity of the images [8]. The Hausdorff distance based algorithms outperform the cross-correlation based methods, especially on images with perturbed pixel locations. Moreover, the algorithms are desired to compare partial images when changes have taken place in the scene. That is, differences of some extent will not lead to severe errors in image registration. Partial Hausdorff distance and voting logic can be used to address this issue.

In most practical systems, computational cost must be considered, especially when the real-time processing is needed. The computational complexity mainly comes from two aspects: large volume of image data and high dimensionality of transformation space. Various search strategies such as multi-resolution methods [9-10] and decomposition of transformation space can be used to reduce computations and hence speed the image alignment process.

In the last few years, local features have become increasingly popular in various applications, such as wide baseline matching, object recognition, image mosaicking, to name just a few domains. In spite of their recent success, local features have a long history. Image matching using a set of local interest points can be traced back to the work of Moravec [11]. The Moravec detector was improved by Harris and Stephens to make it more repeatable under small image variations and near edges [12]. Harris et al. showed its applications in motion tracking, and the Harris corner detector has since widely used in many image matching tasks. While these feature detectors are usually called corner detectors, they are not selecting just corners, but rather any image locations that have large gradients in a few directions in a predefined scale.

Zhang et al. showed that it was possible to match Harris corners over a large image range by using a correlation window around each corner to select likely matches [13]. Schmid et al. showed that invariant local feature matching could be extended to general image recognition problems in which a feature was matched against a large database of images [14]. They also used Harris detector to select interest points, but rather than matching a correlation window, they used a rotationally invariant descriptor of the local image region. This allowed features to be matched under arbitrary orientation change between the two images.

More recently, Lowe proposed a method for extracting distinctive invariant features from images that can be used to perform reliable matching between different views of an object or scene [15]. The method is based on local 3D extrema in the scale-space pyramid built with difference-of-Gaussian filters. The features are invariant to image scale and rotation, and are shown to provide robust matching under considerable geometrical distortion and noise. The recognition approach can robustly 
identify objects among clutter and occlusion while achieving near real-time performance.

In this paper, we deal with such an image registration problem, in which the images were acquired at different times and even by different imaging devices, and changes of some extent may take place in the scene in between the two imaging time. We suppose that the images can be aligned by similarity transform. An efficient and robust algorithm is proposed to solve this problem, which simulates behaviors of human vision in image registration.

By simulating human vision behaviors, we use a two-stage process to perform image registration. First, the rotation angle is computed by comparing the distributions of gradient orientations, which is implemented by a simple 1D correlation. Second, a novel approach is presented to detect significant corners in two images, and the correspondences are established between corners in two images. At this time, the orientation difference has been removed, so the corner correspondences can be established more efficiently. To account for false corner correspondences, the voting method is adopted to determine the transformation parameters, that is, the parameters supported by more corner correspondences are accepted as the true ones.

The rest of this paper is organized as follows. In section 2, we describe the image registration problem and main ideas of the algorithm. Section 3 shows how to compute the rotation angle. Section 4 introduces a novel corner detector. Experimental results are shown in section 5, and some remarks are given in the end.

\section{Problem Formulation and Main Ideas}

Given two images to be registered, one of which is called the reference image and the other is called the sensed image, the two images are denoted by $I$ and $M$, respectively. Mathematically, image registration problem can be formulated as: search a geometric transformation $g$ and an intensity transformation function $f$, so that

$$
I(x, y)=f(M(g(x, y)) .
$$

In most cases, the goal of image registration is aligning two images geometrically (spatial registration), hence the intensity transformation is not always necessary. In some cases, searching such intensity transformation is even impossible, especially in the case of multi-sensor image registration. For this kind of problems, all work of image registration amounts to determining the geometric transformation function $g$, that is, computing the function $g$ so that the sensed image can be spatially registered with the reference image as good as possible. The transformation may be translation, rotation, scaling, similarity transformation, or other more complex transformations such as affine and perspective transformations. In this paper, we only consider similarity transformation, which is defined as follows.

$$
\left(\begin{array}{l}
x^{\prime} \\
y^{\prime}
\end{array}\right)=\left(\begin{array}{l}
t_{x} \\
t_{y}
\end{array}\right)+s\left(\begin{array}{cc}
\cos \theta & \sin \theta \\
-\sin \theta & \cos \theta
\end{array}\right)\left(\begin{array}{l}
x \\
y
\end{array}\right) .
$$


In this case, the problem equals to computing the four parameters, which is denoted by $T=\left(t_{x}, t_{y}, s, \theta\right)$ in the following sections.

As mentioned earlier, it is the structure features such as edges, corners, line segments and boundaries, not the intensity values of the original images that robust image registration relies on. If edge is selected as feature, we can perform edge detection on the two images, then compare the two images in feature space (i.e., point sets) and search the transformation space to determine the parameters that can best match the sensed image with the reference image using the partial Hausdorff distance or other similarity measures. This approach is robust in the sense that it works well under noise, illumination variation and changes of some extent in the scene. But this kind of methods are computationally demanding, especially for high-dimensional transformation spaces such as similarity, affine and perspective transformations. To reduce computations, distance transform and other techniques are used. However, the computational costs are still high.

In many applications, images are rich with line segments resulting from man-made structures, as shown in Fig. 1. In such cases, human vision first determines the orientation difference between two images and then register these images by establishing correspondences of significant features, such as line segments and/or corners, and pays no attention to other features.
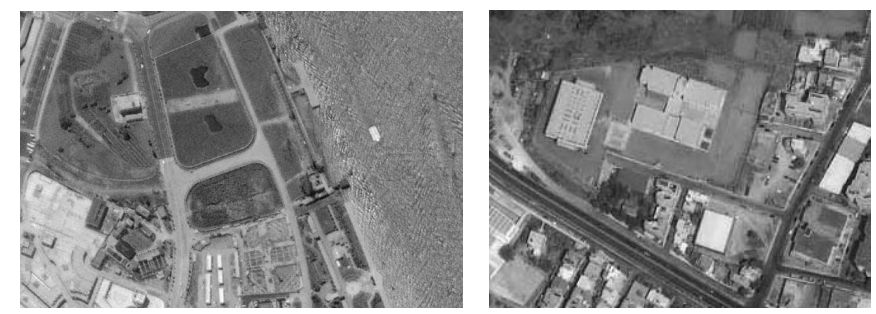

Fig. 1. Images rich with line segments resulting from man-made structures

By simulating human vision behaviors, we perform image registration through a two-stage process. In the first stage, we determine the rotation angle using only the information about the distributions of line orientations in the images, and the process is a simple 1D correlation. The underlying principles of this process are the properties of Hough transform under similarity transformation. To improve the efficiency, we use the distributions of gradient orientations to compute the rotation angle in practice. In this way, the straight line Hough transform, which is of high computational cost, is not necessary. In the second stage, a novel approach is used to detect salient corners in images, and then the transformation parameters can be computed by establishing the correspondences among the corners in two images. Note that the orientation difference has been removed between two images at this time, so the correspondences can be established more efficiently. To account for false corner correspondences, the voting method is adopted to determine the transformation parameters. For our problem, two pairs of corresponding corners uniquely determine the geometric transformation, i.e. one point in the transformation space. The point in transformation 
space, i.e. the transformation parameters, voted for by more corner correspondences is accepted as the solution of the image registration problem.

\section{Computing the Rotation Angle}

The straight line Hough transform(SLHT) is a well-known method for the detection of lines in binary images, and it is also used to locate, recognize some special shape and register images [16-18]. Let a line $L$ be represented by

$$
\rho=x \cos \theta+y \sin \theta \text {. }
$$

Then this line corresponds to point $(\rho, \theta)$ in the Hough space. In the discrete domain, the SLHT transform of an image is a 2D array, in which each row corresponds to one value of $\rho$, and each column to one value of $\theta$. By simple mathematics, it is obvious that this array has the following three properties:

- Rotation in the image plane corresponds to circular shifting of the array;

- If the image is translated, then the same offset value is added to all points in the same column;

- Scaling the image equals to only scaling every $\rho$ in the array, whereas the orientation remains unchanged.

From these properties, it follows that the change of line orientation under similarity transformation is just circular shifting of the array in Hough space. That is, circular shifting of the array is only related to rotation, and independent of translation and scaling. Utilizing this fact, we can compute the rotation angle in the Hough space.

Because Hough transform is computationally demanding, we hope to avoid computing the Hough transform. We know that, if a straight line passes through an edge point, then the gradient orientation at this point is perpendicular to the line with high probability. So, the distributions of gradient orientations in images will behave similarly as the Hough transform. For robustness under noise, the distributions of gradient orientations are computed with the gradient magnitudes as weights. In fact, the distribution is just weighted orientation histogram. Now, the distribution of gradient orientations can be defined as:

$$
h_{i}=\sum_{(x, y)} \operatorname{mag}(x, y) f_{i}(\phi(x, y)) .
$$

Where the sum is over all points of the image, $\operatorname{mag}(x, y)$ is the gradient magnitude, and $f_{i}(\phi(x, y))$ is a function of the gradient orientation $\phi(x, y) . f_{i}(\phi(x, y))=1$ if $\phi(x, y)$ in the specified orientation interval; otherwise, $f_{i}(\phi(x, y))=0$.

In practice, $[0, \pi)$ is uniformly decomposed into a number of intervals according to the expected resolution, then the distribution is computed by (4). If we have 


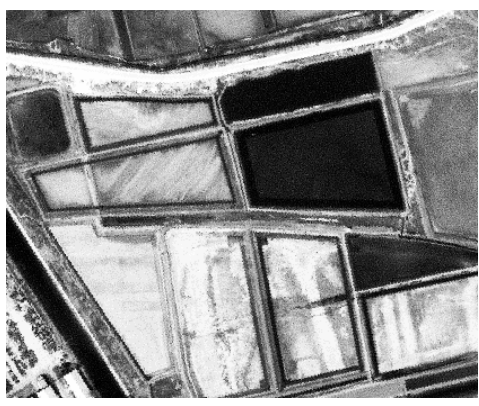

(a)

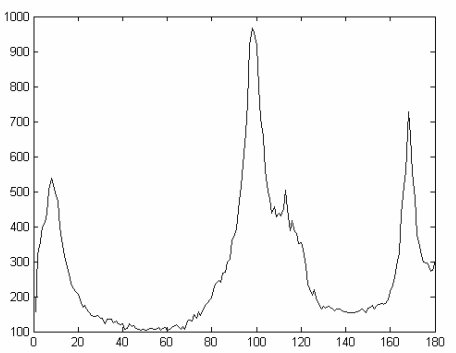

(c)

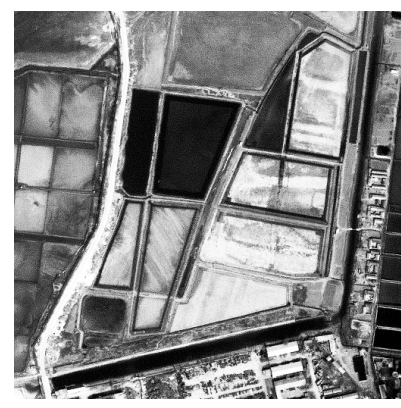

(b)

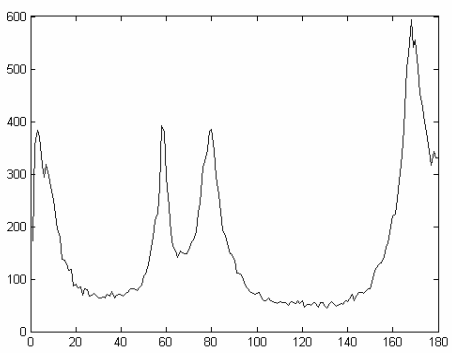

(d)

Fig. 2. An example for computing the rotation angle using gradient orientation distributions. (a) and (b): two images to be registered; (c) and (d): gradient orientation distributions of (a) and (b), respectively. Comparing the two distributions gives the rotation angle, $70^{\circ}$ in this example.

obtained the distributions of two images, then comparing the two distributions gives the rotation angle $\alpha$. Note that the distributions are periodic in $\pi$, so the true rotation angle may be $\alpha$ or $\pi+\alpha$. An example is shown in Fig. 2 .

\section{Corner Detection for Image Registration}

The success of feature-based image registration algorithms depends considerably on effective feature detection. That is, we must select good features and detect the features with effective and efficient algorithms. Good features should have the properties such as repeatability, locality, distinctiveness, accuracy and efficiency. That is, given two images of the same scene, taken under different imaging conditions and some changes in the scene, a high percentage of the features must be detected in both images, whereas only a small number of features detected in one image are not detected in the other image. It is possible only when the features are local enough to limit the risk of a feature including an occluded part and/or parts corresponding to different objects or surfaces, and thus to allow some variations resulting from different imaging conditions and changes in the scene. Because the feature correspondences between two images need to be established, features must be distinctive so that they can be distinguished and matched. 
Corner is increasingly popular in object recognition and image matching. With respect to its practical applications, the corner detection is studied intensively for approximately three decades, and a number of corner detectors have been proposed. For locality and other reasons, these detectors only consider small neighborhoods. But in a small neighborhood, all the measurements would not be precise and reliable enough mainly due to noise. In other words, no detector can make a correct decision whether or not a candidate is a corner without seeing the neighborhood that is big enough. In the image registration applications, images may be acquired under different imaging conditions, at different times, and/or by different devices. So in this case, it is very difficult to ensure the repeatability of corners among images.

To satisfy the requirement of image registration, we propose a novel algorithm for robust corner detection. For a candidate point to be a true corner, there must exist enough points within its neighborhood to support it. The point that is relevant and can make contribution must satisfy two conditions: the gradient magnitude should be large enough, and the gradient orientation should be approximately perpendicular to the line passing through this point and the candidate point. This idea is shown in Fig. 3. By combining the gradient orientation and magnitude, the new approach can consider a large neighborhood and in the meanwhile, exclude most clutters and irrelevant features nearby. For each candidate point, the approach computes three values, which are defined as follows.

- Average Orientation:

$$
\mu_{\phi}=\frac{\sum_{P \in \Omega} f(\varphi(P), \phi(P)) \operatorname{mag}(P) \phi(P)}{\sum_{P \in \Omega} f(\varphi(P), \phi(P)) \operatorname{mag}(P)} .
$$

Where $\Omega$ is neighborhood of point $\mathrm{O} ; \varphi(P)$ and $\phi(P)$ are the orientation of OP and the gradient orientation at point $\mathrm{P}$, respectively; $f(\varphi(P), \phi(P))$ is a function of the two orientations. For example, we can let this function take 1 if one orientation is approximately perpendicular to the other, and 0 if otherwise.

- Orientation Variation:

$$
\sigma_{\phi}^{2}=\frac{\sum_{P \in \Omega} f(\varphi(P), \phi(P)) \operatorname{mag}(P)\left[\phi(P)-\mu_{\phi}\right]^{2}}{\sum_{P \in \Omega} f(\varphi(P), \phi(P)) \operatorname{mag}(P)} .
$$

- Corner Strength:

$$
C S=\sum_{P \in \Omega} f(\varphi(P), \phi(P)) \operatorname{mag}(P) .
$$

In practice, the gradient magnitude mag and orientation $\phi$ are first computed, and all points with the gradient magnitude bigger than a predefined threshold are considered to be candidates for corners. The candidates are then examined by 
computing the values of $\mu_{\phi}, \sigma_{\phi}^{2}$ and $C S$. The candidate with $C S$ as a local maximum and $\sigma_{\phi}^{2}$ greater than chosen threshold is accepted as a corner.
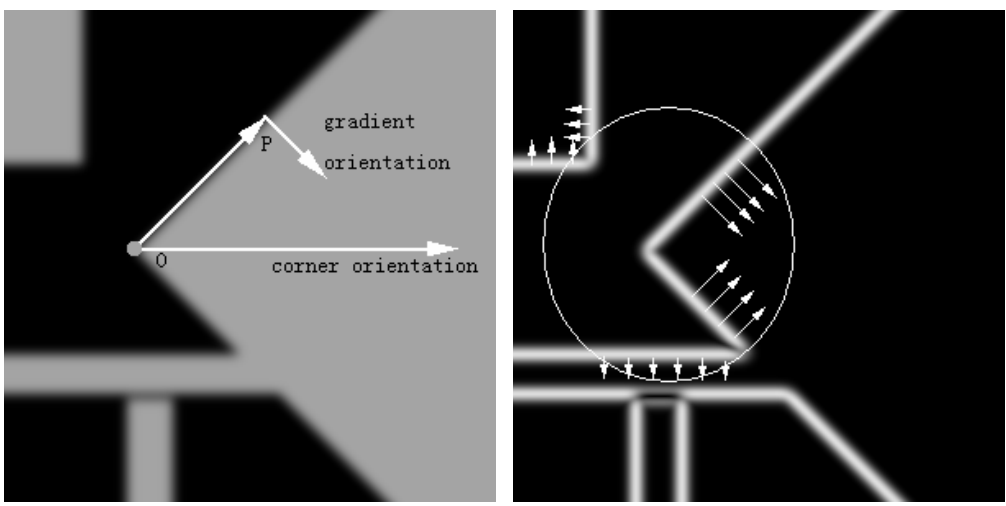

Fig. 3. Ideas for robust corner detection. Irrelevant points in the neighborhood of the candidate corner will be excluded using the gradient orientation information, though these points have high gradient magnitude. Left: original image; right: image for gradient magnitude.

\section{Experimental Results}

Many experiments have been conducted to demonstrate the robustness and efficiency of the presented algorithm using real-world images. Limited by the space, only one experimental result is given in Fig. 4. Here, the reference images were taken by the satellite borne imaging sensors and have been geometrically rectified; the sensed images have been taken by a TV camera mounted on the unmanned aerial vehicle.

The algorithm works well in our experiments. Though there exist significant differences between two images in imaging conditions, modality of sensor and the scene being imaged, both determination of the rotation angle and corner detection are all reliable and robust. For $640 \mathrm{X} 480$ images, an experiment can be carried out within a fraction of second on a $\mathrm{P} 43.0 \mathrm{GHz} \mathrm{PC}$ machine.

\section{Concluding Remarks}

This work has proposed an efficient and robust image registration algorithm by simulating human vision behaviors. The approach is a two-stage process. Based on the properties of Hough transform under similarity transformation, the orientation difference is first removed between two images by a simple $1 \mathrm{D}$ correlation of the gradient orientation distributions; then a novel corner detector is used to extract salient corners, and the transformation parameters can be computed by establishing corner correspondences between the two images. In future work, more complex geometric transformations will be dealt with by generalizing the ideas presented in this paper. 


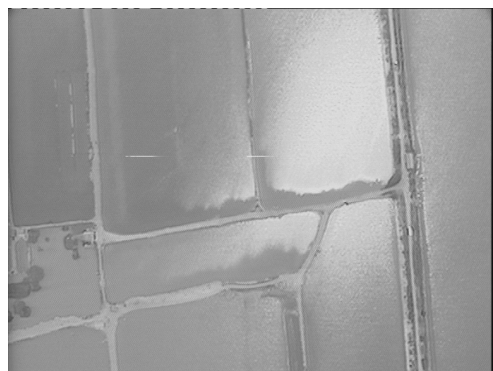

(a) the sensed image

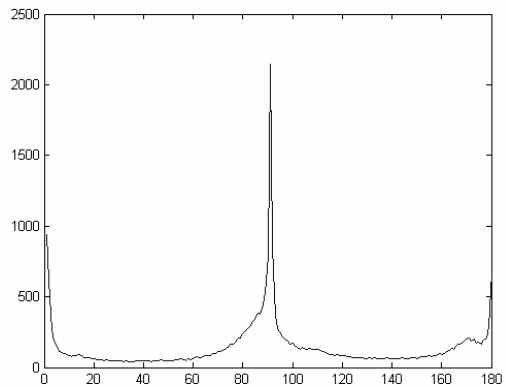

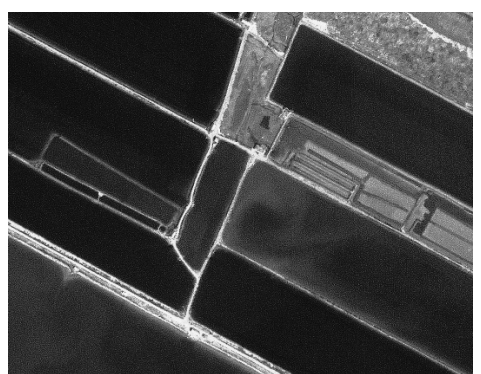

(b) the reference image

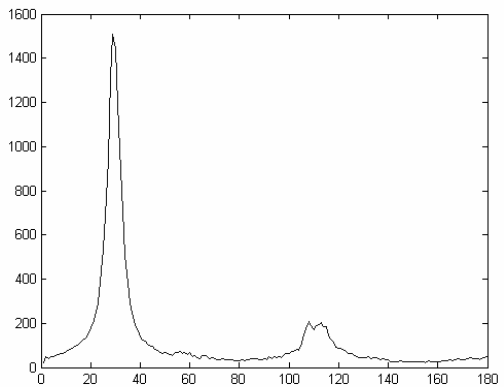

(c) the gradient orientation distribution of (a) (d) the gradient orientation distribution of (b)

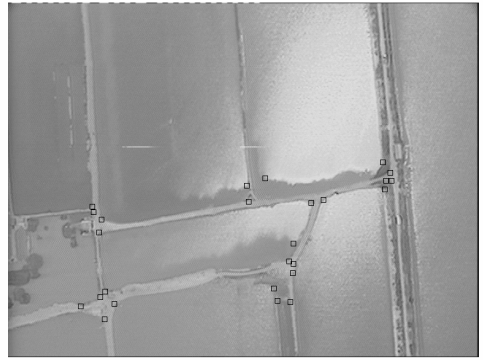

(e) result of corner detection on (a)

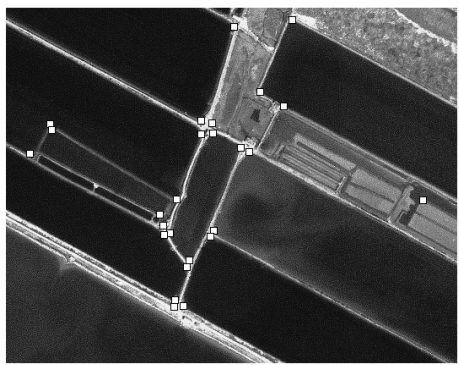

(f) result of corner detection on (b)

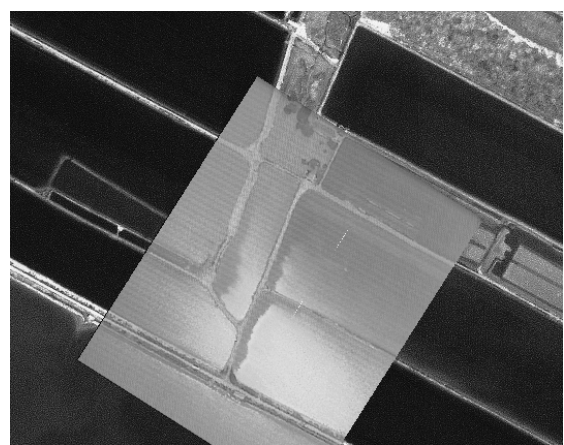

(g) result of image registration

Fig. 4. Experimental results using the image registration algorithm presented in this paper 


\section{References}

1. Brown, L.G.: A Survey of Image Registration Techniques. ACM Computing Surveys 24, 226-376 (1992)

2. Zitova, B., Flusser, J.: Image Registration Methods: A Survey. Image and Vision Computing 21, 977-1000 (2003)

3. Goshtasby, A., Stockman, G.C., Page, C.V.: A Region-Based Approach to Digital Image Registration with Subpixel Accuracy. IEEE Trans. on Geoscience and Remote Sensing 24, 390-399 (1986)

4. Alhichri, H.S., Kamel, M.: Virtual circles: a new set of features for fast image registration. Pattern Recognition Letters 24, 1181-1190 (2003)

5. Moss, S., Hancock, E.R.: Multiple Line-Template Matching with EM Algorithm. Pattern Recognition Letters 18, 1283-1292 (1997)

6. Habib, A.F., Alruzouq, R.I.: Line-based modified iterated Hough transform for automatic registration of multi-source imagery. The Photogrammetric Record 105, 5-21 (2004)

7. Stockman, G., Kopstein, S., Benett, S.: Matching Images to Models for Registration and Object Detection via Clustering. IEEE Trans. on Pattern Analysis and Machine Intelligence 4, 229-241 (1982)

8. Huttenlocher, D.P., Klanderman, G.A., Rucklidge, W.J.: Comparing Images Using the Hausdorff Distance. IEEE Trans. on Pattern Analysis and Machine Intelligence 15, 850 863 (1993)

9. Zheng, Q., Chellapa, R.: A computational vision approach to image registration. IEEE Trans. on Image Processing 2, 311-325 (1993)

10. Borgefors, G.: Hierarchical chamfer matching: a parametric edge matching algorithm. IEEE Trans. on Pattern Analysis and Machine Intelligence 10, 849-865 (1988)

11. Moravec, H.: Rover Visual Obstacle Avoidance. In: The Seventh International Joint Conference on Artificial Intelligence, IJCAI 1981, Vancouver, Canada, pp. 785-790 (1981)

12. Harris, C., Stephens, M.: A Combined Corner and Edge Detector. In: The Fourth Elvey Vision Conference, Manchester, UK, pp. 147-151 (1988)

13. Zhang, Z., Deriche, R., Faugeras, O., Luong, Q.T.: A Robust Technique for Matching Two Uncalibrated Images Through the Recovery of the Unknown Epipolar Geometry. Artificial Intelligence 78, 87-119 (1995)

14. Schmid, C., Mohr, R.: Local Grayvalue Invariants for Image Retrieval. IEEE Trans on Pattern Analysis and Machine Intelligence 19, 530-535 (1997)

15. Lowe, D.G.: Distinctive Image Features from Scale-Invariant Keypoints. International Journal of Computer Vision 60(2), 91-110 (2004)

16. Ballard, D.H.: Generalizing the Hough Transform to Detect Arbitrary Shapes. Pattern Recognition 13, 111-122 (1981)

17. Illingworth, J., Kittler, J.: A Survey of the Hough Transform. Graphics and Image Processing 44, 87-116 (1988)

18. Pao, D.C.W., Li, H.F., Jayakumar, R.: Shapes Recognition Using the Straight Line Hough Transform: Theory and Generalization. IEEE Trans. on Pattern analysis and Machine Intelligence 14, 1076-1089 (1992) 